\title{
Análise do desenvolvimento neuropsicomotor de pré-termos em ambulatório multidisciplinar: um olhar da fisioterapia
}

\section{Analysis of neuropsychomotor development of preterm in multidisciplinary ambulatory: a view of physiotherapy}

\author{
Nicole Almeida ${ }^{1}$ (1) \\ Daiane Aparecida da Silva ${ }^{2}$ (1) \\ Larissa Rebola Volpi da Silva ${ }^{3}$ (1)
}

Audrin Said Vojciechowski
Arlete Ana Motter
Talita Gianello Gnoato Zotz

1,2,5Universidade Federal do Paraná (Curitiba).Paraná, Brasil.nicolealmeida854@gmail.com, daiane.silvaa97@gmail.com, arlete.motter@uol.com.br , ${ }^{3}$ Complexo Hospital de Clínicas da Universidade Federal do Paraná (Curitiba). Paraná, Brasil. larissa_ela@hotmail.com ${ }^{4}$ Universidade Federal do Paraná (Curitiba), Faculdade UniBras (Juazeiro). Paraná, Bahia, Brasil. asaidvoj@gmail.com ${ }^{6}$ Autora para correspondência. Universidade Federal do Paraná (Curitiba). Paraná, Brasil. talita.gnoato@gmail.com

\begin{abstract}
RESUMO | INTRODUÇÃO: Desde a gestação até o nascimento, o desenvolvimento neuropsicomotor é influenciado por diversos fatores biopsicossociais. Tal influência pode ser ainda mais acentuada em recém-nascidos pré-termo devido a internação na Unidade de Terapia Intensiva Neonatal, por isto, bebês pré-termos estão mais susceptíveis a essas influências. OBJETIVO: Analisar sob a ótica do fisioterapeuta o desenvolvimento neuropsicomotor de pré-termos em ambulatório multidisciplinar de um hospital público. METODOLOGIA: Estudo observacional e longitudinal, ambispectivo, de 19 crianças nascidas prematuras de 0 a 12 meses de idade corrigida, sendo critério de exclusão crianças com alterações cognitivas e/ou genéticas ou não terem assinado o termo de consentimento. Os dados foram coletados por meio da análise de prontuários entre agosto de 2017 e dezembro de 2018, o desenvolvimento avaliado por meio do Teste Triagem Denver II, em dois momentos: A1 e A2, em sua admissão e retorno ao serviço após a alta da Unidade de Terapia Intensiva Neonatal e para análise da relação entre mãe e bebê foi utilizada a Avaliação Vínculo Mãe/filho apenas em A1. As estimulações eram realizadas por meio de orientações e folder educativo para os pais e responsáveis. Os dados foram analisados, por meio de média, desviopadrão, frequência absoluta e relativa, mediana, minimo e máximo e Teste de Wilcoxon para os domínios do Teste Denver II A1 em comparação a A2, utilizando o programa SPSS versão 20.0. RESULTADOS: Foram acompanhadas 19 crianças, destas $79 \%$ foram classificadas com desenvolvimento normal no A1. As crianças que apresentaram déficits no A1 $(10,5 \%)$, superaram no A2. Porém, no segundo atendimento, algumas passaram a apresentar dificuldade em novas tarefas/domínio (motor, motor fino e pessoal-social), de modo que $31,5 \%$ apresentaram desenvolvimento geral anormal em A2. Foi encontrada diferença significativa $(p=0,02)$ ao comparar as classificações gerais de Denver II nos dois momentos de avaliação. O vínculo mãe-filho foi classificado como fraco (média de 5,8 pontos). CONCLUSÃo: Observou-se que os bebês superaram os atrasos identificados na primeira avaliação, embora os resultados no Denver tiveram variações ao longo do tempo. Dessa forma, acredita-se que o perfil familiar identificado nesta pesquisa somada a abordagem multiprofissional possa ter favorecido o desenvolvimento neuropsicomotor dos bebês prematuros com até 12 meses de idade corrigida.
\end{abstract}

PALAVRAS-CHAVE: Recém-Nascido Prematuro. Fisioterapia. Desenvolvimento infantil. Estimulação precoce.
ABSTRACT | INTRODUCTION: From gestation to birth, neuropsychomotor development is influenced by several biopsychosocial factors. This influence can be even more accentuated in preterm newborns due to their admission to the Neonatal Intensive Care Unit, so preterm babies are more susceptible to these influences. OBJECTIVE: To analyze the neuropsychomotor development of preterm babies in a multidisciplinary outpatient clinic of a public hospital. METHODOLOGY: Observational and longitudinal, ambispective study of 19 children born prematurely from 0 to 12 months of corrected age, being exclusion criteria children with cognitive and/or genetic alterations or not having signed the consent form. The data were collected through the analysis of medical records between August 2017 and December 2018, the development evaluated through the Denver II Triage Test, in two moments: $\mathrm{A} 1$ and $\mathrm{A} 2$, on their admission and return to service after discharge from the Neonatal Intensive Care Unit and for analysis of the relationship between mother and baby the Evaluation Mother/Son bond only in A1 was used. The stimulations were carried out employing orientation and educational folder for parents and guardians. The data were analyzed by means of mean, standard deviation, absolute and relative frequency, median, minimum and maximum, and Wilcoxon Test for the domains of Denver II A1 compared to A2, using the SPSS program version 20.0. RESULTS: 19 children were accompanied, and $79 \%$ of these were classified with normal development in A1. The children who presented deficits in A1 (10.5\%), surpassed in A2. However, in the second attendance, some started to present difficulties in new tasks/domains (motor, fine motor, and personal-social), so that $31.5 \%$ presented abnormal general development in A2. A significant value was found when comparing the Denver II general classification in A1 with A2 $(p=0.02)$. The mother-child bond was classified as weak (average of 5.8 points). CONCLUSION: It was observed that babies overcame the delays identified in the first evaluation, although the results in Denver had variations over time. Thus, it is believed that the family profile identified in this research plus the multi-professional approach may have favored the neuropsychomotor development of premature babies up to 12 months of corrected age.

KEYWORDS: Premature Newborn. Physiotherapy. Child Development. Early Stimulation. 


\section{Introdução}

O desenvolvimento infantil é influenciado por fatores biológicos, ambientais, sociais e culturais. Este processo é explicado pela abordagem biopsicossocial que leva em consideração a interação entre as condições de saúde e os fatores do contexto biológico e ambiental ${ }^{1}$. Associado a isso, considera-se a Teoria dos Sistemas Dinâmicos, dentro dos diferentes sistemas presentes no organismo, sejam eles mecânicos ou de estímulos sensórios do ambiente, suas pequenas partes possuem determinância sobre o todo, e, com isto, o movimento pode emergir como resultado da interação entre os diferentes elementos, sem um controle ou padrão advindo do sistema nervoso².

Considerando isso, crianças nascidas pré-termo possuem maior risco de apresentarem atrasos motores, pois além da imaturidade fisiológica, também são expostas a dor repetitiva, ventilação mecânica, procedimentos invasivos e excesso de estímulos ambientais inadequados, decorrentes da longa permanência destes em UTIs Neonatais (UTIN) $)^{3}$. Além destes fatores, outros como o baixo peso ao nascer, a hospitalização precoce e prolongada, asfixia perinatal, apneia, infecções, icterícia, hemorragias intracranianas são fatores de risco para atrasos do desenvolvimento infantil ${ }^{3}$.

Após a alta hospitalar, também é preciso considerar os riscos ambientais para o desenvolvimento motor que a criança encontra, como o espaço físico em que a criança vive, a escolaridade dos pais, a dinâmica familiar, o poder aquisitivo da família, as relações familiares e o vínculo materno ${ }^{4}$. Em relação ao vínculo mãe-filho, devido ao parto prematuro, é interrompido abruptamente, pois o recém-nascido necessitará de cuidados intensivos em ambiente hospitalar ${ }^{5}$.

Por conta de todos esses fatores, é fundamental que os prematuros sejam avaliados no âmbito das alterações motoras, neurológicas e comportamentais para a detecção precoce de possíveis dificuldades e consequentemente o fisioterapeuta irá delinear uma intervenção precoce que contemple o maior número possível de fatores biopsicossociais.
Sabe-se que programas de intervenção precoce para bebês prematuros têm influência positiva no desenvolvimento cognitivo e motores durante a infância. Neste sentido, o Ministério da Saúde, definiu metas para a estimulação precoce, considerando tanto o ambiente ambulatorial quanto o ambiente familiar, viabilizando a atuação multiprofissional. Além disso, sugere-se orientação aos pais e à comunidade a respeito de acompanhamento contínuo desde o nascimento até a idade escolar ${ }^{6}$.

Os recentes avanços nas áreas de obstetrícia e neonatologia são responsáveis pelo aumento da taxa de sobrevivência de nascimentos pré-termo ${ }^{7}$, de modo que Curitiba e região metropolitana concentram 30\% dos nascimentos do estado do Paraná, dos quais 9,9\% nascem antes de 37 semanas de gestação ${ }^{8}$. Além disso, crianças pré-termo que sobrevivem possuem maior risco de morbidades a curto e longo prazo ${ }^{3}$.

Portanto, é necessário realizar a avaliação do DNPM e acompanhamento deste, a fim de identificar potenciais riscos, atrasos ou melhoras, para que estratégias para prevenir e/ou minimizar atrasos no desenvolvimento motor desses prematuros, por meio da instrumentalização familiar, possam ser identificadas.

Assim, o objetivo do estudo foi analisar sob a ótica do fisioterapeuta o desenvolvimento neuropsicomotor ao longo dos 12 primeiros meses de idade corrigida de pré-termos acompanhados em ambulatório multidisciplinar.

\section{Metodologia}

O presente estudo, de caráter observacional, ambispectivo e de acompanhamento longitudinal durante o primeiro ano, sendo período total do estudo de 2017 a 2019, aprovado pelo Comitê de Ética em Pesquisa em Seres Humanos do HC-UFPR, parecer 2.330.736, CAAE 58865616.7.0000.0096, foi realizado com crianças prematuras pós alta da Unidade de Terapia Intensiva Neonatal. 
Os acompanhamentos foram realizados em um Ambulatório de Puericultura, de um Hospital Público de Curitiba. A coleta de dados ocorreu entre agosto de 2017 e dezembro de 2018. As famílias realizavam o acompanhamento multidisciplinar a cada 3 meses. No qual o desenvolvimento do bebê era avaliado/reavaliado por uma equipe de médicos neuropediatras, fisioterapeuta, assistente social, terapeuta ocupacional e psicólogos.

A amostra foi composta por bebês prematuros, com 0 a 12 meses de idade corrigida, pré-termos, após alta da UTIN. Foram excluídos da amostra: bebês que apresentavam lesão no sistema nervoso central, alterações genéticas, que tenham passado por procedimentos cirúrgicos abdominais, e/ou os responsáveis tenham se recusado a assinar o Termo de Consentimento Livre Esclarecido. Os responsáveis também precisavam se comprometer com a dinâmica do serviço prestado, uma vez que necessitavam passar a tarde toda em atendimento e também realizar as orientações passadas pela equipe de saúde. Caso não houvesse possibilidade/interesse em dar continuidade, a família era encaminhada para outro ambulatório de puericultura (Figura 1).

Figura 1. Desenho do Estudo

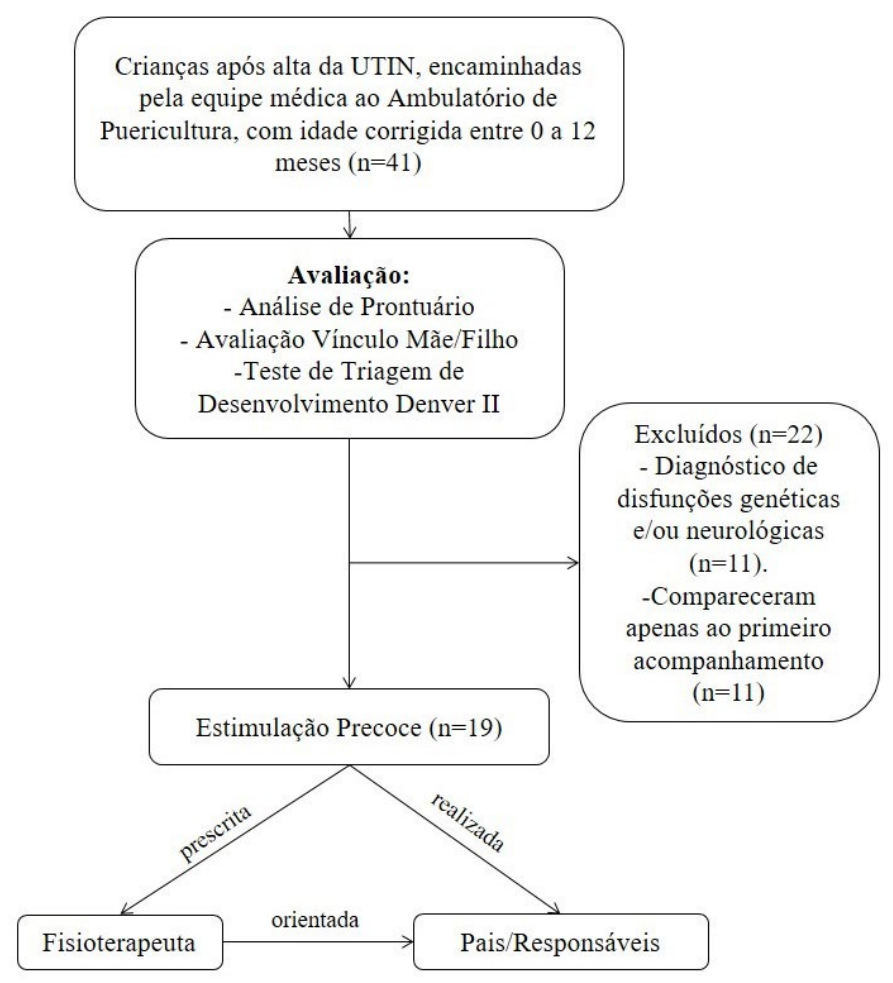

Os atendimentos realizados pela equipe multidisciplinar e observados na presente pesquisa eram norteados pela coleta de dados do prontuário referente ao período de internamento e considerações sociodemográficas da família, além disso, foram aplicados alguns protocolos para avaliação do desenvolvimento neuropsicomotor, biopsíquico e do vínculo materno. Dessa forma, eram realizados os seguintes testes: Teste de Triagem de Denver II (TTDII)ำ, Avaliação do vínculo mãe/filho ${ }^{10}$.

As variáveis de caracterização dos recém-nascidos foram: idade gestacional, tipo de parto, índice de Apgar no primeiro e no quinto minuto de vida, peso e altura ao nascer, tempo de internação na UTIN, dias de uso de: ventilação mecânica, pressão positiva contínua nas vias aéreas (CPAP), ventilação por pressão positiva intermitente nasal (NIPPV) e/ou máscara de oxigênio e presença de: icterícia, sepse, apneia, hipotonia, acidose metabólica, síndrome do desconforto respiratório, infecção do trato urinário e mau estado geral ao nascer (MEG). Também foram coletados os dados maternos e paternos como idade, escolaridade, ocupação principal, estado civil, local de residência. 
Para classificar o peso ao nascer, foram considerados recém-nascidos com baixo peso aqueles com peso inferior a $2500 \mathrm{~g}$, muito baixo peso aqueles que apresentaram peso ao nascer menor que $1500 \mathrm{~g}$ e extremo baixo peso aqueles com peso ao nascimento abaixo de $1000 \mathrm{~g}^{11}$.

O Teste de Triagem de Desenvolvimento Denver II avalia crianças de zero a seis anos com um total de 125 itens divididos em quatro aspectos: pessoal-social - com enfoque na socialização da criança; motricidade fina-englobando a coordenação olho-mão; motricidade grossa-que inclui o controle motor corporal; e linguagem-compreendendo a capacidade de reconhecimento, entendimento e uso da linguagem. Os itens avaliados foram categorizados de forma individual, considerando a idade cronológica e a idade corrigida de cada criança. O resultado pode ser expresso de três formas: com atraso - quando um item é intersectado pela linha da idade após o percentil 90 e este não é realizado pela criança. Cautela ou atenção - quando a criança não cumpre um item que a linha da idade se encontra entre 75 e 90\%, ou então passou - quando a criança realizava a prova com sucesso. O desempenho final foi classificado de acordo com o número de itens em atraso e/ou cautela. Considerou-se o desenvolvimento: anormal - quando se obteve dois ou mais atrasos, independente da área; questionável - se apresentado um atraso ou duas ou mais cautelas; e normal - se não apresentado nenhum atraso, ou no máximo uma cautela ${ }^{9}$.

A Avaliação Vínculo Mãe/filho utilizada para avaliar o vínculo criança-cuidador, composta por 13 perguntas com respostas do tipo sim/não, sendo "sim" resposta positiva à presença de um atributo ou indicador de fraco vínculo. Somando-se as respostas "sim", obtémse um escore que pode variar de 1 a 13. A classificação positiva para fraco vínculo se dá com número de respostas positivas $\geq 5^{10}$.

A avaliação inicial (A1) da criança era composta por: análise do prontuário, aplicação da Avaliação Vínculo Mãe/filho10 e primeira avaliação do Teste de Triagem Denver IIํ. Após 3 meses, esta criança retornava ao serviço para uma reavaliação (A2), composta apenas pelo Teste de Triagem Denver II, neste dia, novas orientações eram passadas à família, de acordo com a idade corrigida que a criança se encontrava.
A criança era avaliada e, em seguida, demonstravase aos pais quais estímulos deveriam ser realizados em domicílio de acordo com a idade (estímulos para controle cervical, rolar, sentar, posição de gatas ou ficar em pé/caminhar), e entregava-se folders construídos pela equipe fisioterapêutica com estas mesmas orientações para dar suporte.

O poder da amostra foi calculado para o $\mathrm{n}$ de 19 crianças no programa G*Power3.1.3, considerando os seguintes critérios: tamanho do efeito:0,50; erro a:0,05, resultando no poder (1- $\beta$ ) de 0,85 . Os dados foram analisados por meio da média, desviopadrão, frequência absoluta e relativa, mediana, mínimo e máximo. Por meio do teste de Wilcoxon, foi realizada a comparação entre a Classificação geral do Teste de Denver II no A1 com A2., para isso foi utilizado o programa SPSS (Statistical Package for Social 18 Sciences) versão 20.0.

\section{Resultados}

Haviam 41 crianças nascidas pré-termo, com idade corrigida entre 0 a 12 meses, após alta da Unidade de Terapia Intensiva Neonatal elegíveis para o estudo. Considerando os critérios de exclusão, 22 crianças não entraram no estudo, devido ao não seguimento das famílias ao ambulatório $(n=11)$ e devido a disfunções neurológicas e/ou genéticas $(n=11)$, portanto a amostra final foi de 19 crianças.

A Tabela 1 apresenta dados de caracterização da amostra sobre dados neonatais. O tempo médio de permanência na UTIN foi de 48,7 dias, $84,2 \%$ das crianças necessitavam CPAP e $63,1 \%$ apresentaram quadro de icterícia. As intercorrências materno-infantis foram: trabalho de parto prematuro sem motivo, diabetes mellitus gestacional $(21 \% ; n=4)$, Síndrome Hipertensiva Gestacional $(63,1 \% ; n=12)$, pós operatório de cirurgia bariátrica $(10,5 \% ; n=2)$, descolamento prematuro da placenta $(10,5 \% ; n=2)$, centralização fetal $(5,25 \% ; n=1)$, sífilis congênita materna $(5,25 \% ; n=1)$, restrição de crescimento intrauterino $(10,5 \% ; n=2)$, redução do líquido amniótico $(5,25 \% ; n=1)$, miomatose uterina $(5,25 \% ; n=1)$, ruptura prematura de membranas $(5,25 \% ; n=1)$, bolsa rota $(5,25 \% ; n=1)$, infecção urinária $(5,25 \% ; n=1)$, placenta prévia total $(5,25 \% ; n=1)$. 


\begin{tabular}{|c|c|}
\hline Tempo de gestação (semanas) & $29,9 \pm 2,47$ \\
\hline \multicolumn{2}{|l|}{ Tipo do parto } \\
\hline Normal & $31,6 \%(n=6)$ \\
\hline Cesária & $68,4 \%(n=13)$ \\
\hline \multicolumn{2}{|l|}{ Apgar 1} \\
\hline Abaixo de 7 & $42,2 \%(n=8)$ \\
\hline Igual ou Acima de 7 & $57,8 \%(n=11)$ \\
\hline \multicolumn{2}{|l|}{ Apgar $5^{-}$} \\
\hline Abaixo de 7 & $10,6 \%(n=2)$ \\
\hline Igual ou Acima de 7 & $89,4 \%(n=17)$ \\
\hline \multicolumn{2}{|l|}{ Apgar $10^{\circ}$} \\
\hline Abaixo de 7 & - \\
\hline Igual ou Acima de 7 & $10,5 \%(n=2)$ \\
\hline Peso ao nascer (gramas) & $1251,5 \pm 506,5$ \\
\hline \multicolumn{2}{|l|}{ Classificação } \\
\hline Baixo Peso & $15,7 \%(n=3)$ \\
\hline Muito Baixo Peso & $57,8 \%(n=11)$ \\
\hline Extremo Baixo Peso & $21 \%(n=4)$ \\
\hline Altura ao nascer $(\mathrm{cm})$ & $37,7 \pm 4,7$ \\
\hline Perímetro cefálico $(\mathrm{cm})$ & $27,3 \pm 2,9$ \\
\hline Tempo de permanência na UTIN (dias) & $48,7 \pm 30,5$ \\
\hline Ventilação Mecânica (SIMV) & $36,8 \%(n=7)$ \\
\hline CPAP & $84,2 \%(n=16)$ \\
\hline NIPPV & $10,6 \%(n=2)$ \\
\hline Máscara de Oxigênio & $36,8 \%(n=7)$ \\
\hline Icterícia & $63,1 \%(n=12)$ \\
\hline Síndrome do Desconforto Respiratório & $10,5 \%(n=2)$ \\
\hline Sepse neonatal & $15,8 \%(n=3)$ \\
\hline Apneia de prematuridade & $36,8 \%(n=7)$ \\
\hline Mau estado geral ao nascer & $31,5 \%(n=6)$ \\
\hline Acidose Metabólica & $10,5 \%(n=2)$ \\
\hline Infecção do trato Urinário & $5,2 \%(n=1)$ \\
\hline Hipotonia ao nascer & $10,5 \%(n=2)$ \\
\hline Cianótico & $15,8 \%(n=3)$ \\
\hline
\end{tabular}


A Tabela 2 apresenta a classificação de cada aspecto do Teste de Denver II.

Tabela 2. Comparação do desempenho no Teste de Denver II A1 com o A2 ( $n=19)$

\begin{tabular}{|c|c|c|}
\hline & $A 1(n=19)$ & $A 2(n=19)$ \\
\hline \multicolumn{3}{|l|}{ Idade em meses } \\
\hline Média, DV & $3,6 \pm 1,4$ & $7,7 \pm 1,9$ \\
\hline Mediana (min; max) & $3,1 \quad(2,3 ; 8,3)$ & $7,4(5,1 ; 11,3)$ \\
\hline \multicolumn{3}{|l|}{ Domínio motor } \\
\hline Normal & $89,5 \%(n=17)$ & $63,2 \%(n=12)$ \\
\hline Questionável & $5,2 \%(n=1)$ & $21 \%(n=4)$ \\
\hline Anormal & $5,2 \%(n=1)$ & $15,8 \%(n=3)$ \\
\hline \multicolumn{3}{|l|}{ Domínio linguagem } \\
\hline Normal & $100 \%(n=19)$ & $89,5 \%(n=17)$ \\
\hline Questionável & 0 & $10,5 \%(n=2)$ \\
\hline Anormal & 0 & 0 \\
\hline \multicolumn{3}{|l|}{ Domínio motor fino } \\
\hline Normal & $89,5 \%(n=17)$ & $89,5 \%(n=17)$ \\
\hline Questionável & $10,5 \%(n=2)$ & 0 \\
\hline Anormal & 0 & $10,5 \%(n=2)$ \\
\hline \multicolumn{3}{|l|}{ Domínio Pessoal-social } \\
\hline Normal & $94,8 \%(n=18)$ & $84,3 \%(n=16)$ \\
\hline Questionável & $5,2 \%(n=1)$ & $10,5 \%(n=2)$ \\
\hline Anormal & 0 & $5,2 \%(n=1)$ \\
\hline \multicolumn{3}{|l|}{ Classificação Denver } \\
\hline Normal & $79 \%(n=15)$ & $42,2 \%(n=8)$ \\
\hline Questionável & $10,5 \%(n=2)$ & $26,3 \%(n=5)^{*}$ \\
\hline Anormal & $10,5 \%(n=2)$ & $31,5 \%(n=6)^{*}$ \\
\hline
\end{tabular}

$\mathrm{A} 1=\overline{\text { Acompanhamento } 1 ; \mathrm{A} 2=\text { Acompanhamento } 2 ;{ }^{*} \text { valor significativo quando comparado } a}$ Classificação Denver A1 e A2 ( $p=0.02$, Wilcoxon).

A Tabela 3 apresenta dados socioeconômicos e perfil familiar da amostra. Dos dados sociodemográficos, pode-se notar que as mães apresentam média de idade de 27,3 anos, mostrando um perfil de mães jovens, em $89,4 \%$ das famílias, os pais possuem estado civil casado. Das mães, 52,6\% apresentavam ensino médio completo, enquanto os pais, $63,1 \%$, apresentavam ensino médio completo, $52,6 \%$ possuíam moradia própria, $78,9 \%$ moravam em Curitiba e $84,2 \%$ na zona urbana. 
Tabela 3. Dados sociodemográficos dos pais de prematuros $(n=19)$

\begin{tabular}{|c|c|}
\hline & média $\pm D P ; \%(n)$ \\
\hline Vínculo Mãe Filho & $5,8 \pm 2,8$ \\
\hline Idade Materna & $27,3 \pm 5,5$ \\
\hline \multicolumn{2}{|l|}{ Estado civil Materna } \\
\hline Solteira & $10,5 \%(n=2)$ \\
\hline Casada & $89,5 \%(n=17)$ \\
\hline \multicolumn{2}{|l|}{ Escolaridade Materna } \\
\hline Analfabeta & $0 \%(n=0)$ \\
\hline Fundamental Incompleto & $0 \%(n=0)$ \\
\hline Fundamental Completo & $5,2 \%(n=1)$ \\
\hline Médio Incompleto & $21 \%(n=4)$ \\
\hline Médio Completo & $52,6 \%(n=10)$ \\
\hline Superior Incompleto & $5,2 \%(n=1)$ \\
\hline Superior Completo & $15,8 \%(n=3)$ \\
\hline Pré-Natais & $52,6 \%(n=10)$ \\
\hline Idade Paterna & $30,3 \pm 8,1$ \\
\hline \multicolumn{2}{|l|}{ Estado civil Paterno } \\
\hline Solteiro & $10,5 \%(n=2)$ \\
\hline Casado & $89,5 \%(n=17)$ \\
\hline \multicolumn{2}{|l|}{ Escolaridade Paterna } \\
\hline Analfabeta & $0 \%(n=0)$ \\
\hline Fundamental Incompleto & $5,2 \%(n=1)$ \\
\hline Fundamental Completo & $5,2 \%(n=1)$ \\
\hline Médio Incompleto & $0 \%(n=0)$ \\
\hline Médio Completo & $63,1 \%(n=12)$ \\
\hline Superior Incompleto & $5,2 \%(n=1)$ \\
\hline Superior Completo & $21 \%(n=4)$ \\
\hline
\end{tabular}

\section{Discussão}

Analisando sob a ótica do fisioterapeuta o desenvolvimento neuropsicomotor ao longo dos 12 primeiros meses de idade corrigida dos pré-termos acompanhados no ambulatório, verificou-se que houve prevalência de crianças com prematuridade acentuada. No que diz respeito aos responsáveis legais, a maior parte das famílias participantes eram compostas pelo pai e pela mãe, os quais possuíam o ensino médio completo.

No presente estudo, associado à orientação dos pais, utilizou-se como suporte educacional cartilhas desenvolvidas pela equipe fisioterapêutica, norteando o trabalho a ser realizado em casa. É imprescindível a instrumentalização aos responsáveis, já que a orientação adequada para que estes saibam das dificuldades, limitações e das diferenças pessoais de ritmo e de potencial da criança a ser estimulada de modo que haja um sincronismo entre pais e terapeuta ${ }^{12}$. Estudo ${ }^{13}$ que também fez uso deste tipo instrumento de apoio aos pais apresentou resultado positivo ao longo do desenvolvimento infantil. Ressalta-se que, mesmo com uso destes, as estimulações eram definidas de acordo com a necessidade de cada criança.

Apesar de todas estimulações orientadas pela fisioterapeuta serem de caráter motor, e considerando que no grupo multiprofissional não havia fonoaudióloga, foi possível constatar que crianças com atraso no domínio linguagem e pessoal-social superaram os déficits. Acredita-se que os pais, ao realizarem o estímulo motor em seus filhos, proporcionaram também estímulos a este domínio, seja por meio de canções, falas, conversas ou até mesmo mantendo o contato visual com a criança. 
O fato de algumas crianças possuírem oscilações na classificação do desenvolvimento pode ser atribuído ao fato de que a plasticidade do cérebro em desenvolvimento pode estar limitada devido à prematuridade, trazendo consequências à aquisição de habilidades. Para Giacchini e colaboradores $\frac{12}{}$, a identificação precoce das situações que levam à lesão cerebral, a prescrição adequada da conduta em cada caso, e a utilização de fatores de proteção neuronal podem influir positivamente em cada caso, o que reforça de aproveitar precocemente as aberturas terapêuticas, possibilitando maiores resultados relacionados à plasticidade cerebral. Observa-se que todas as crianças que apresentaram questionamentos ou atrasos em itens da Denver em um dos acompanhamentos superaram estes na avaliação seguinte. Esta oscilação na classificação enfatiza a necessidade de acompanhamento contínuo desta população, corroborando com Araújo e colaboradores ${ }^{13}$.

Embora o número da amostra deste estudo tenha sido pequeno, foi possível observar que a estimulação precoce realizada pelos pais, que foram previamente orientados e auxiliados com material impresso, fez com que o desenvolvimento neuropsicomotor evoluísse positivamente. Ressalta-se a necessidade de acompanhamento periódico do desenvolvimento motor de bebês prematuros.

O parto prematuro traz consigo a vulnerabilidade, dependência e necessidade de proteção do prematuro, interferindo no vínculo mãe-filho ${ }^{5}$, uma vez que a separação abrupta desta díade gera medo e insegurança na mãe. Esta separação precoce atrasa a responsabilidade materna pelos cuidados do filho, gerando assim um sentimento de "não se sentir maternal", aumentando a influência negativa sobre o vínculo desta díade ${ }^{5}$. Além disso, estudos encontraram relação significativa entre desenvolvimento motor e interação mãe-filho ${ }^{14}$. Por meio da Avaliação Vínculo Mãe-Filho pode-se constatar que houve fraco vínculo entre as díades estudadas. Cabe ressaltar que tal fato pode ser explicado pois a média de semanas do parto foi de 29,9 semanas.

Assim como o vínculo materno, a presença paterna tem sua importância no desenvolvimento infantil, uma vez que o pai tem papel protetor à criança, além de prestar apoio financeiro e psicológico à mãe ${ }^{4}$. Pilz e Schermann ${ }^{1}$ constataram, por meio do Teste Denver II, que crianças cujas mães não possuíam o apoio paterno, a probabilidade de apresentarem suspeita de atraso no desenvolvimento neuropsicomotor era sete vezes maior.

O grau de escolaridade materna é outro fator que pode interferir no desenvolvimento neuropsicomotor. Em estudo epidemiológico brasileiro ${ }^{15}$, entre os anos de 2007 e 2016, as maiores taxas de prematuridade estavam relacionadas ao menor tempo de escolaridade materna (até três de anos de estudo). Além disso, estudos apontam que este dado sociodemográfico é um indicador de reconhecimento e acesso aos serviços de saúde, portanto quanto maior a escolaridade, melhor o entendimento da necessidade de cuidados especiais durante a gestação e maior o fator de proteção para o desenvolvimento ${ }^{16}$.

Além dos fatores ambientais e gestacionais, citados anteriormente, que apresentam risco ao desenvolvimento neuropsicomotor, também é necessário considerar os eventos neonatais. O Ministério da Saúde ${ }^{17}$ considera que recém-nascido de risco é toda criança que apresenta, pelo menos, um critério dos que se seguem: residência em área de risco; peso ao nascer menor que $2.500 \mathrm{~g}$; recém-nascido com menos de 37 semanas de gestação; Apgar $<7$ no $5^{\circ}$ minuto de vida; hospitalização ou intercorrência na maternidade ou unidade de assistência ao recém-nascido; necessidade de orientação especial na alta da maternidade; filho de mãe adolescente ( $<18$ anos); mãe com baixa instrução ( $\leq 8$ anos de estudo); história de morte de criança menor de 5 anos na família. Portanto, a amostra estudada apresenta pelo menos dois indicadores de risco, sendo eles a prematuridade e a necessidade de assistência após o nascimento.

Com relação às características neonatais ao nascimento, os dados do presente estudo corroboram com Oliveira e colaboradores ${ }^{7}$, apontando que a maior parte dos nascidos prematuros apresentam escore maior que 7 na avaliação de Apgar no 50 minuto, não apresentando risco neurológico. Ainda considerando os critérios de risco ao recém-nascido, o baixo peso associado à prematuridade é diretamente proporcional ao risco de morbidade ${ }^{18}$. Além disso, sabe-se que prematuros com baixo peso apresentam maior risco de atraso no desenvolvimento motor no primeiro ano de vida, podendo se estender para idades mais avançadas ${ }^{19}$. Estudo $\underline{7}$ aponta forte associação do peso ao nascer com nascimentos prematuros, corroborando com o presente estudo, em que $94,7 \%$ da amostra nasceu com peso inferior a $2.500 \mathrm{~g}$. 
Também há de se considerar o tempo médio de permanência na UTIN, o que influencia no desenvolvimento neuropsicomotor ${ }^{3,19}$. Em estudo realizado na cidade de São Paulo, em que os bebês foram separados em dois grupos com tempo de internação acima ou abaixo de 34 dias de internamento, constatou-se que o grupo com maior permanência na UTIN apresentou atraso no desenvolvimento motor, enquanto o outro grupo encontrava-se adequado para a idade corrigida.

A literatura, muitas vezes, relaciona a prematuridade a apenas alguns fatores de risco ao desenvolvimento neuropsicomotor, tais como baixo peso ao nascer, tempo de hospitalização, características maternas. Porém, é preciso considerar a Teoria dos Sistemas Dinâmicos 2 . Desta forma, o presente estudo não isolou fatores biológicos e/ou ambientais, por considerar que há muitos fatores que influenciarão no desenvolvimento neuropsicomotor.

As Diretrizes de Estimulação Precoce para Crianças de zero a 3 anos com Atraso no Desenvolvimento Neuropsicomotor ${ }^{6}$ recomendam que as equipes que realizarão a estimulação precoce desenvolvam, em conjunto com a criança e sua família, uma intervenção exclusiva, levando em consideração avaliações multidisciplinares que visam a autonomia e a independência do usuário, bem como sua inclusão social.

A intervenção motora precoce proporciona uma grande quantidade de estímulos novos para a criança. A qualidade do dia a dia do bebê, de que forma ele é cuidado, e as influências geradas pelo seu meio também serão muito importantes para a qualidade e o sucesso do desenvolvimento da criança, assim como orientações para os pais também são necessárias. $O$ fisioterapeuta tem o papel de conduzir a avaliação inicial e contínua da criança, desenvolvendo metas e objetivos através da utilização de métodos adequados às suas necessidades, como a instrumentalização da família ${ }^{20}$.

O número amostral, o tempo de acompanhamento, a grande quantidade de fatores que influenciam o desenvolvimento motor são limitações intrínsecas às pesquisas que estudam o desenvolvimento de bebês pré-termos.

\section{Conclusão}

Em relação ao desenvolvimento neuropsicomotor dos bebês acompanhados, observou-se que estes superaram os atrasos identificados na primeira avaliação. Dessa forma, acredita-se que o perfil familiar identificado nesta pesquisa somada às implicações clínicas relacionadas à abordagem multiprofissional e cartilhas de orientações para desenvolvimento motor elaboradas pela equipe fisioterapêutica possam ter favorecido o desenvolvimento neuropsicomotor dos bebês prematuros com até 12 meses de idade corrigida, todavia sugere-se mais estudos sobre a temática.

\section{Agradecimentos}

As fisioterapeutas Marise Bueno Zonta, Marcela Fischer Almeida, Luzanira Correia Feitosa, Karize Rafaela Mesquita Novakoski, Michele Aparecida Marques, Roberta Maria Alvares, Larissa Rebola Volpi da Silva e Isamar Villa Carvalho que elaboraram os folderes de orientações aos pais para estimulação do desenvolvimento neuropsicomotor dos bebês que frequentam o serviço.

\section{Contribuições dos autores}

Almeida N, Silva DA, Silva LRV, Vojciechowski AS, Motter AA e Zotz TGG participaram da concepção, delineamento, busca e análise estatística dos dados da pesquisa, interpretação dos resultados e redação do artigo científico.

\section{Conflitos de interesses}

Nenhum conflito financeiro, legal ou político envolvendo terceiros (governo, empresas e fundações privadas, etc.) foi declarado para nenhum aspecto do trabalho submetido (incluindo, mas não se limitando a subvenções e financiamentos, participação em conselho consultivo, desenho de estudo, preparação de manuscrito, análise estatística, etc.).

\section{Referências}

1. Pilz EML, Schermann LB. Determinantes biológicos e ambientais no desenvolvimento neuropsicomotor em uma amostra de crianças de Canoas/RS. Ciên saúde coletiva. 2007;12(1):182-90. http://dx.doi.org/10.1590/S1413-81232007000100021

2. Camargos ACR, Lacerda TTB. O Desenvolvimento motor na perspectiva dos sistemas dinâmicos. Temas desenvolv [Internet]. 2005;14(82):23-9. Disponível em: https://pesquisa.bvsalud.org/ portal/resource/pt/lil-533191 
3. Giachetta L, Nicolau CM, Costa APMB, Zuana AD. Influência do tempo de hospitalização sobre o desenvolvimento neuromotor de recém-nascidos pré-termo. Fisioter. pesqui. 2010;17(1):24-9. http://dx.doi.org/10.1590/S1809-29502010000100005

4. Araujo LB, Mélo TR, Israel VL. Baixo peso ao nascer, renda familiar e ausência do pai como fatores de risco ao desenvolvimento neuropsicomotor. J. Hum. Growth Dev. 2017;27(3):272-80. http://dx.doi.org/10.7322/jhgd.124072

5. Spinelli M, Frigerio A, Montali L, Fasolo M, Spada MS, Mangili G. 'I still have difficulties feeling like a mother': the transition to motherhood of preterm infants mothers. J Health Psychol. 2016;31(2):184-204. https://doi.org/10.1080/08870446.2015.1088 $\underline{015}$

6. Ministério da Saúde (Brasil). Secretaria De Atenção À Saúde. Diretrizes de estimulação precoce: crianças de zero a 3 anos com atraso no desenvolvimento neuropsicomotor decorrente de microcefalia [Internet]. Brasília: Ministério da Saúde; 2016. Disponível em: https://bvsms.saude.gov.br/bvs/publicacoes/ diretrizes estimulacao criancas 0a3anos neuropsicomotor.pdf

7. Oliveira LL, Gonçalves AC, Costa JSD, Bonilha ALL. Fatores maternos e neonatais relacionados à prematuridade. Rev. esc. enferm. USP. 2016;50(3):382-9. http://dx.doi.org/10.1590/S0080623420160000400002

8. Ministério da Saúde (Brasil). SINASC-Sistema de Informações de Nascidos Vivos [Internet]. Brasília: Ministério da Saúde; 2017. [citado em 2019 jul. 30]. Disponível em: http://tabnet.datasus.gov. br/cgi/deftohtm.exe?sinasc/cnv/nvuf.def

9. Sabatés AL, Lamônica DAC, Perissinoto J, Brêtas JS, Silva MGB, Rezende MA, Resegue RFS, Isotani SM. Teste de triagem do desenvolvimento Denver II: adaptação transcultural para a criança brasileira. Com autorização do autor Frankenburg WK. São Paulo, 2013.

10. Mader CV, Monteiro VL, Spada PV, Nóbrega FJ. Avaliação do vínculo mãe-filho e saúde mental de mães de crianças com deficiência intelectual. Einstein (São Paulo). 2013;11(1):63-70. http://dx.doi.org/10.1590/S1679-45082013000100012

11. Caçola P, Bobbio TG. Baixo peso ao nascer e alterações no desenvolvimento motor: a realidade atual. Revista Paulista de Pediatria. 2010;28(1):70-76. https://doi.org/10.1590/S010305822010000100012
12. Giacchini V, Tonial A, Mota HB. Aspectos de linguagem e motricidade oral observados em crianças atendidas em um setor de estimulação precoce. Distúrb Comum [Internet]. 2013;25(2):253-65. Disponível em: https://revistas.pucsp.br/index. php/dic/article/view/16478/12375

13. Araujo DM, Ribeiro MF, Espíndula AP. Treino materno para estimulação domiciliar sugere melhora no desenvolvimento motor de prematuros. ConsSaúde. 2015;14(3):385-93. https://doi. org/10.5585/conssaude.v14n3.5665

14. Soares H, Barbieri-Figueiredo M, Pereira P, Silva M, Fuertes $M$. Parents attending to nurse visits and birth age contribute to infant development: a study about the determinants of infant development. Early Hum. Dev. 2018;122:15-21. https://doi. org/10.1016/j.earlhumdev.2018.05.006

15. Santos RJ. Prematuridade no Brasil: um estudo epidemiológico no período de 2007 a 2016. [trabalho de conclusão de curso] [Internet]. Vitória de Santo Antão: Universidade Federal de Pernambuco; 2018. Disponível em: https://www.repositorio.ufpe. br/bitstream/123456789/28884/1/Santos\%2c\%20Robervaldo\%20 Jos\%c3\%a9\%20dos.pdf

16. Ramos HAC, Cuman RKN. Fatores de risco para prematuridade: pesquisa documental. Esc. Anna Nery. 2009;13(2):297-304. http://dx.doi.org/10.1590/S1414$\underline{81452009000200009}$

17. Ministério da Saúde (Brasil). Secretaria de Atenção à Saúde, Departamento de ações programáticas estratégicas. Agenda de Compromissos para a Saúde Integral da Criança e Redução da Mortalidade Infantil [Internet]. Brasília: Ministério da Saúde; 2005. Disponível em: http://bvsms.saude.gov.br/bvs/publicacoes/ agenda_compro_crianca.pdf

18. Passini R, Cecatti JG, Lajos GJ, Tedesco RP, Nomura ML, Dias $\mathrm{T}$, et al. Brazilian multicentre study on preterm birth (EMIP): prevalence and factors associated with spontaneous preterm birth. Plos One. 2014;9(10):1-12. https://doi.org/10.1371/journal. pone. 0109069

19. Saccani R, Valentini NC, Pereira KR, Müller AB, Gabbard C. Associations of biological factors and affordances in the home with infant motor development. Pediatr Int. 2013;55(2):197-203. Citado em: PMID: 23279095

20. Silva CCV. Atuação da fisioterapia através da estimulação precoce em bebês prematuros. Rev. Eletrôn Atualiza Saúde [Internet]. 2017;5(5):29-36. Disponível em: http://atualizarevista. com.br/article/atuacao-da-fisioterapia-atraves-da-estimulacaoprecoce-em-bebes-prematuros/ 